University of Wollongong

Research Online

Australian Institute for Innovative Materials -

Papers

Australian Institute for Innovative Materials

2013

Magnetotransport dependence on the field magnitude and direction in large area epitaxial graphene film on stretchable substrates

Peite Bao

University of Sydney

Wenxian Li

University of Wollongong, wenxian@uow.edu.au

Wai Kong Yeoh

University of Sydney

Xiangyuan Cui

University of Sydney

J. H. Kim

University of Wollongong, jhk@uow.edu.au

See next page for additional authors

Follow this and additional works at: https://ro.uow.edu.au/aiimpapers

Part of the Engineering Commons, and the Physical Sciences and Mathematics Commons

Research Online is the open access institutional repository for the University of Wollongong. For further information contact the UOW Library: research-pubs@uow.edu.au 


\title{
Magnetotransport dependence on the field magnitude and direction in large area epitaxial graphene film on stretchable substrates
}

\author{
Abstract \\ We report the magnetotransport properties of large area graphene on stretchable polyethylene \\ terephthalate substrates. At $2 \mathrm{~K}$, weak localization of electrons introduced negative magnetoresistance at \\ low field; a transition to positive magnetoresistance followed as the external field increases. Our results \\ suggest that weak localization contributes to Hall effect at low temperature. At room temperature, only \\ classical Lorentz force contribution can be observed. Angular dependence of the external magnetic field \\ on longitudinal and transverse resistivity is measured to test the interplay between weak localization and \\ Lorentz force contribution. Quantitative simulations based on quantum interference theory produced \\ excellent agreement with the experiments.

\section{Disciplines} \\ Engineering | Physical Sciences and Mathematics
}

\section{Authors}

Peite Bao, Wenxian Li, Wai Kong Yeoh, Xiangyuan Cui, J. H. Kim, Yong-Mook Kang, Wenrong Yang, S X. Dou, Simon P. Ringer, and Rongkun Zheng 


\title{
Magnetotransport dependence on the field magnitude and direction in large area epitaxial graphene film on stretchable substrates
}

\author{
Peite Bao, ${ }^{1}$ Wenxian $\mathrm{Li},{ }^{2,3}$ Wai Kong Yeoh, ${ }^{4}$ Xiangyuan Cui, ${ }^{4}$ Jung Ho Kim, ${ }^{2}$ \\ Yong-Mook Kang, ${ }^{5}$ Wenrong Yang, ${ }^{6}$ Shi Xue Dou, ${ }^{2}$ Simon P. Ringer, ${ }^{4}$ \\ and Rongkun Zheng 1 a) \\ ${ }^{1}$ School of Physics, The University of Sydney, New South Wales 2006, Australia \\ ${ }^{2}$ Institute for Superconducting and Electronic Materials, The University of Wollongong, \\ New South Wales 2522, Australia \\ ${ }^{3}$ School of Computing, Engineering and Mathematics, The University of Western Sydney, \\ Penrith South, NSW 2751, Australia \\ ${ }^{4}$ Australian Centre for Microscopy and Microanalysis, The University of Sydney, New South Wales 2006, \\ Australia and School of Aerospace, Mechanical and Mechatronic Engineering, The University of Sydney, \\ New South Wales 2006, Australia \\ ${ }^{5}$ Department of Chemistry, Dongguk University, Pildong-3ga, Jung-gu, Seoul 100-715, South Korea \\ ${ }^{6}$ School Life and Environmental Science, Deakin University, Geelong, Vic 3217, Australia
}

(Received 20 August 2012; accepted 25 February 2013; published online 7 March 2013)

\begin{abstract}
We report the magnetotransport properties of large area graphene on stretchable polyethylene terephthalate substrates. At $2 \mathrm{~K}$, weak localization of electrons introduced negative magnetoresistance at low field; a transition to positive magnetoresistance followed as the external field increases. Our results suggest that weak localization contributes to Hall effect at low temperature. At room temperature, only classical Lorentz force contribution can be observed. Angular dependence of the external magnetic field on longitudinal and transverse resistivity is measured to test the interplay between weak localization and Lorentz force contribution. Quantitative simulations based on quantum interference theory produced excellent agreement with the experiments. C 2013 American Institute of Physics. [http://dx.doi.org/10.1063/1.4794868]
\end{abstract}

Owing to its peculiar band dispersion, graphene exhibits a wealth of anomalous transport phenomena, such as the unconventional quantum Hall effect, Klein tunneling, and weak localization (WL) and weak antilocalization (WAL) of electrons. ${ }^{1-5}$ All these characters made graphene an ideal candidate in a wide range of nano-devices, such as the flexible and transparent conductive electrodes, high-frequency field effect transistors (FET), and extraordinary magnetoresistance (EMR) devices. $^{6-8}$ Over the past few years, remarkable improvements have been achieved in fabricating and optimizing graphene flakes and ribbons device prototypes, mainly by micromechanical cleaving or exfoliation from natural graphite. ${ }^{9-12}$

Notable differences in transport properties of graphene from other traditional two-dimensional (2D) system, e.g., quantum wells, have been observed. ${ }^{13}$ Magnetotransport properties, like all transport behaviors, are fundamentally associated to the electron scattering and localization. In disordered electronic systems, WL effect manifests a negative magnetoresistance (MR) and hence a WL peak at zero external field. This is due to the external magnetic field that applied onto the 2D graphene system, would add an additional phase to the electron waves, and therefore destroy the destructive interferences. ${ }^{14}$ In single-layered graphene, WL is strongly suppressed whereas it is stored in bilayered and multilayered graphene as results of the interesting competition between WL and WAL effects, which is caused by the relationship between charge carriers in the two valleys of the band structure and its sublattice symmetry. ${ }^{5,15-18}$ The quantitative description of this magnetotransport behavior has been established by using the

a)Electronic mail: rongkun.zheng@sydney.edu.au. quantum interference theory, where the WL MR has been taken as an indication of the quality of the graphene samples. ${ }^{15,19-22}$ At low field region, WL correction to the MC, using the quantum interference theory, can be described as

$$
\begin{gathered}
\Delta \sigma(B)=\frac{e^{2}}{\pi h}\left[F\left(\frac{B}{B_{\phi}}\right)-F\left(\frac{B}{B_{\phi}+2 B_{i}}\right)-2 F\left(\frac{B}{B_{\phi}+B_{*}}\right)\right], \\
F(z)=\ln z+\psi\left(\frac{1}{2}+\frac{1}{z}\right), B_{\phi, i, *}=\frac{\hbar}{4 D e} \tau_{\phi, i, *}^{-1}=\frac{\hbar c}{4 e} L_{\phi, i, *}^{-2},
\end{gathered}
$$

where $\psi(x)$ is the digamma function, $\tau_{\varphi}$ is the decoherence time, $\tau_{i}$ is the inter-valley scattering time, and $\tau_{*}^{-1}=\tau_{\varphi}^{-1}$ $+\tau_{i}^{-1}$. The corresponding characteristic length, $L_{\phi, i, *}$, can be obtained by the fitting the parameters from the equation. ${ }^{16,19}$

On the other hand, obtaining macroscopic graphene sheets is crucial in realizing the proposed graphene-based devices. ${ }^{23}$ Among all the techniques, chemical vapor deposition (CVD), which enables large area graphene sheet patterning with the transfer technique onto the arbitrary substrates, has a huge impact in the fabrication of graphene. ${ }^{24-26} \mathrm{As}$ such, from a fundamental perspective, understanding the magnetotransport behaviors in large area graphene film on different substrates is of obvious importance as it will help its eventual technological applications. This is especially true for epitaxial grown graphene that is very different from the exfoliated ones since the large area graphene films can quite often be considered as self-averaging systems. ${ }^{26-28}$ In this work, we studied the magnetotransport behaviors of a large area graphene sheet up to $\sim 20 \mathrm{~mm}^{2}$ on a stretchable polyethylene terephthalate (PET) substrate. ${ }^{24}$ The longitudinal resistivity 
(a)

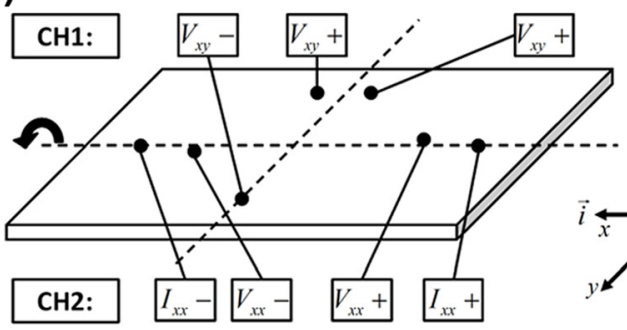

(b)

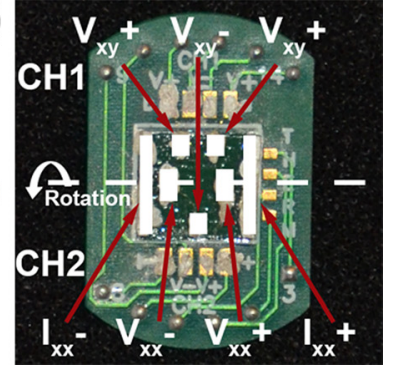

FIG. 1. (a) Schematic diagram showing the configuration of sample wiring for measurements, and (b) an actual image of the testing chip with tested transparent graphene film on PET substrate on top. The testing area is defined by the distance between the voltage detection on $x x$ and $x y$ direction. The arrow on left shows the rotation direction. $\rho_{x x}$ and the transverse resistivity $\rho_{x y}$ show interesting dependence on the magnitude and direction of the external field, which can be reproduced and confirmed by numerical simulations. These results provide insight on field dependence magnetotransport properties, which may have implication for device applications.

Large area graphene film sample was fabricated by CVD method from the same facility as described in Ref. 24 . The rapid cooling in Argon atmosphere during the synthesize process assured that the sample is dominated by graphene bilayers and accompanied with monolayer and multilayer, as confirmed by Raman spectroscopy and scanning electron microscopy. ${ }^{24}$ The total area is up to a few square centimeters. Magnetotransport measurements were carried out on a Quantum Design $^{\text {TM }}$ Physical Property Measurement System (PPMS) with a horizontal sample rotator along the current axis under an external magnetic field up to $1 \mathrm{~T}$, and temperature ranged from $2 \mathrm{~K}$ to $300 \mathrm{~K}$. Figure 1 shows the wiring configuration patterned by silver paint onto a $\sim 20 \mathrm{~mm}^{2}$ area, where a DC excitation current $i=1 \mathrm{~mA}$ current was applied along the $x x$ direction, and $\mathrm{CH} 1$ and $\mathrm{CH} 2$ measured the voltages in $x y$ and $x x$ directions, respectively.

Figures 2(a) and 2(d) shows the raw experimental data of longitudinal resistance, $R_{C H 2}$, under a $-1 \mathrm{~T}$ to $1 \mathrm{~T}$ external field applied normal to the sample plane at $2 \mathrm{~K}$ and $300 \mathrm{~K}$, respectively. These curves are asymmetric, containing mixing contribution of longitudinal and transverse resistance. It is hard to ensure the vectors from $V_{x x}+$ to $V_{x x}-$ and $V_{x y}+$ to
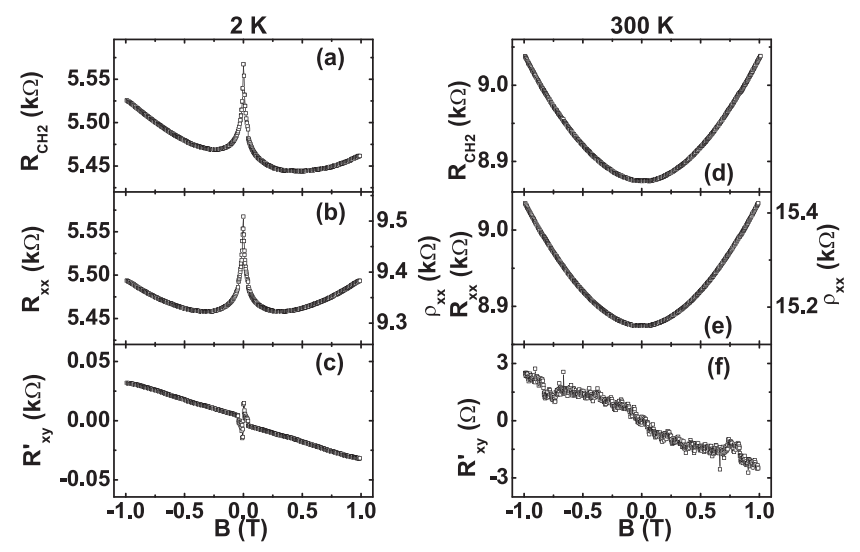

FIG. 2. (a) Raw experimental data of $R_{\mathrm{CH} 2}$ under a scanning external magnetic field normal to the graphene plane at $2 \mathrm{~K}$, and the curve is asymmetry. (b) Corrected longitudinal resistance $R_{x x}$ and resistivity $\rho_{x x}$ at $2 \mathrm{~K}$, where a clear WL peak is seen at zero field. (c) $R_{x y}{ }^{\prime}$ at $2 \mathrm{~K}$, which shows a linear relationship to the magnetic field, indicating that the difference between $R_{C H 2}$ and $R_{x x}$ comes from the Hall resistance. (d) $R_{C H 2}$ at $300 \mathrm{~K}$ from the raw experimental data, and (e) the corrected longitudinal resistance $R_{x x}$ and resistivity $\rho_{x x}$, only positive MR $\propto B^{2}$ is seen. (f) Plots the $R_{x y}{ }^{\prime}$ at $300 \mathrm{~K}$ and also shows a linear relationship to the magnetic field.
$V_{x y}-$ in the wiring configuration are, respectively, parallel to the $x x$ and $x y$ directions. Therefore, either of the two channels reflects the pure longitudinal or transverse resistance, and further data treatment to distinguish the two contributions is required. The components for longitudinal resistance, negative MR due to WL and positive MR due to WAL and Lorentz force contribution, are both even functions of the magnetic field while the Hall resistance is an odd function of the field. Thus, the pure longitudinal resistance $R_{x x}$ and transverse resistance $R_{x y}$ of the sample can be obtained as

$$
\left\{\begin{array}{l}
R_{x x}(B)=\frac{1}{2}\left[R_{C H 2}(B)+R_{C H 2}(-B)\right] \\
R_{x y}(B)=\frac{1}{2}\left[R_{C H 1}(B)-R_{C H 1}(-B)\right],
\end{array}\right.
$$

where $R_{C H 1}(B)$ and $R_{C H 2}(B)$ represent the resistance values under an external field $B$ in the raw data. ${ }^{22}$ Hence, the longitudinal component mixed into $R_{C H 1}(B)$, denoted by $R_{x x}{ }^{\prime}(B)$, and the transverse component mixed into $R_{C H 2}(B)$, denoted by $R_{x y}{ }^{\prime}(B)$, can be determined using $R_{x x}{ }^{\prime}(B)=\frac{1}{2}\left[R_{C H 1}(B)+\right.$ $\left.R_{C H 1}(-B)\right]$ and $R_{x y}{ }^{\prime}(B)=\frac{1}{2}\left[R_{C H 2}(B)-R_{C H 2}(-B)\right]$. Sheet resistivities on the longitudinal and transverse directions can be calculated from $R_{x x}, R_{x y}$ by dividing a factor decided by the dimensions of the sample and therefore have an identical trend of the pure resistance $R_{x x}$ and $R_{x y}$.

The longitudinal resistance $R_{x x}$ as well as the resistivity $\rho_{x x}$ (magnetoresistivity) after data correction at $2 \mathrm{~K}$ and $300 \mathrm{~K}$ are shown in Figures 2(b) and 2(e). At $2 \mathrm{~K}$, a typical negative MR can be observed at field below $\sim \pm 0.3 \mathrm{~T}$, which can be attributed to WL correction to the MR since the external magnetic field destroys the destructive interference, leading to a decrease in the resistance. The cyclic motion of electrons becomes more significant with the increasing field, resulting positive MR $\left(\propto B^{2}\right)$. Consequently, a transition from negative to positive MR can be observed. While at $300 \mathrm{~K}$, only positive MR can be seen at all field range since the quantum interference phenomena was strongly suppressed due to the high temperature. Figures 2(c) and 2(f) plot the $R_{x y}{ }^{\prime}$ at two temperatures, and both show linear relationship to the magnetic field, indicating the residual comes from the Hall resistance. It is also worth noting that we have observed the graduate falling of longitudinal resistivity in certain temperature regions as temperature decreases, which was attributed to different conducting phases. ${ }^{29}$ The similar phenomenon confirms that our tests were all performed at semiconductor phases.

Figures 3(a) and 3(d) show $R_{C H 1}$ at $2 \mathrm{~K}$ and $300 \mathrm{~K}$, respectively. It can be seen that $R_{C H 1}(B=0) \neq 0$ in both curves, due to the longitudinal component. Linear Hall resistance components can be extracted from the raw data by using Eq. (2) 

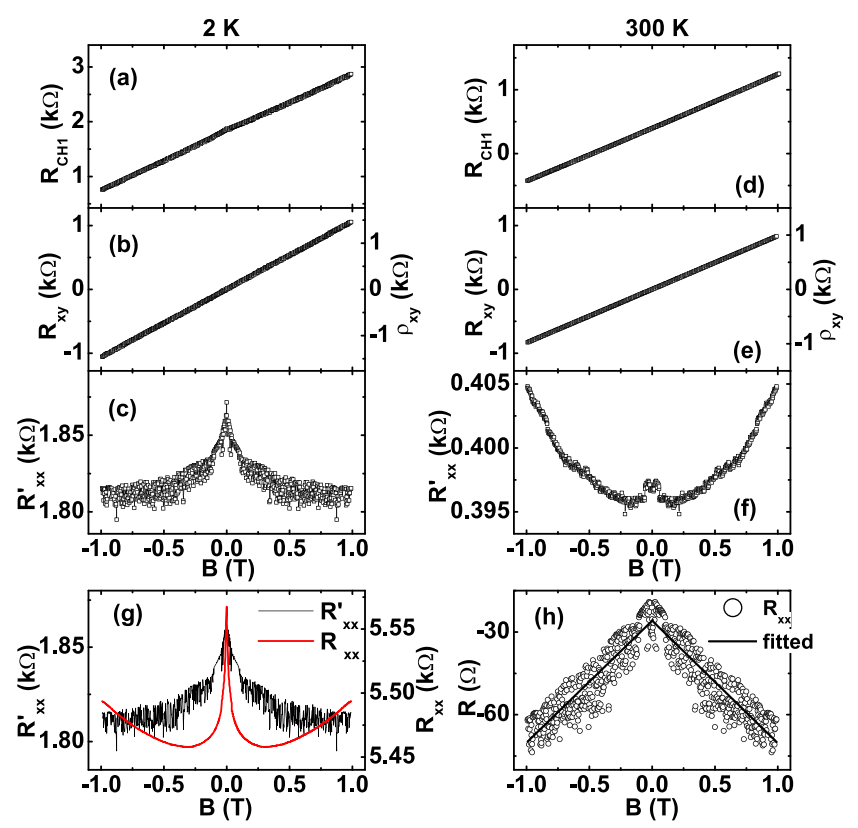

FIG. 3. (a) Raw experimental data of $R_{C H 1}$ under a scanning external magnetic field normal to the graphene plane at $2 \mathrm{~K}$, in which $R_{C H 1}(B=0) \neq 0$. (b) Corrected transverse resistance $R_{x y}$ and resistivity $\rho_{x y}$ at $2 \mathrm{~K}$, showing typical Hall resistivity characteristics. (c) The $R_{x x}{ }^{\prime}$ at $2 \mathrm{~K}$. (d) The $R_{C H 1}$ at $300 \mathrm{~K}$ from the raw experimental data, and (e) the corrected transverse resistance $R_{x y}$ and resistivity $\rho_{x y}$. (f) Plots the $R_{x x}{ }^{\prime}$ at $300 \mathrm{~K}$ showing similar curve to the $R_{x x}$ at the same temperature. (g) Compares the $R_{x x}{ }^{\prime}$ with $R_{x x}$ at $2 \mathrm{~K}$ and (h) shows the difference between and $R_{x x}$, fitted curve suggesting that it has two linear parts.

for both $2 \mathrm{~K}$ and $300 \mathrm{~K}$, as shown in Figures 3(b) and 3(e). The longitudinal components can also be extracted as shown in Figures 3(c) and 3(f). Interestingly, at $2 \mathrm{~K}$, as presented in Figure $3(\mathrm{~g}), R_{x x}{ }^{\prime}$ and $R_{x x}$ show different trends, and the difference after normalization as well as the fitted curve is plotted in the Figure 3(h). It shows a linear relationship to the absolute value of the magnetic field. On the other hand, $R_{x x}{ }^{\prime}$ plotted in Figure 3(f) is in the same quadratic shape of $300 \mathrm{~K} R_{x x}$.

Unlike previous reports on large area graphene films patterned by CVD, longitudinal resistance in this work did not show either (1) obvious conductance fluctuations or (2) an overall monotonous negative magnetoresistance. ${ }^{22,26,27}$ In contrast to the universal conductance fluctuation (UCF) in mesoscopic graphene where sample size is similar to characteristic length, fluctuation in large area/macroscopic graphene is normally attributed to classical reasons, i.e., by large scale inhomogeneity. ${ }^{15,22}$ Therefore the smooth curve, in Figure 2(b), suggests good uniformity of the sample even after been transferred onto PET substrates. Also, it has been concluded by many works that WL can be used as a sensitive probe for judging the quality of the graphene samples. ${ }^{15}$ In few layer graphene, atomically sharp disorder, i.e., defects, may promote WL effect and in some scenarios where heavy defects could result in an overall negative magnetoresistance. ${ }^{27}$ However, our results clearly show a transition from negative to positive magnetoresistance at low $B$, suggesting relatively better sample quality. On the other hand, from the transverse resistance/ Hall effect, the carrier density of our epitaxial graphene film sample is derived to be around $5.92 \times 10^{11} \mathrm{~cm}^{-2}$ at $2 \mathrm{~K}$, and $7.46 \times 10^{11} \mathrm{~cm}^{-2}$ at $300 \mathrm{~K}$, which are comparable to other literatures. $^{23}$ However, the carrier mobility of our sample, $\sim 1.11 \times 10^{3} \mathrm{~cm}^{2} \mathrm{~V}^{-1} \mathrm{~s}^{-1}$ at $2 \mathrm{~K}$ and $5.43 \times 10^{2} \mathrm{~cm}^{2} \mathrm{~V}^{-1} \mathrm{~s}^{-1}$ at
$300 \mathrm{~K}$, is considerably lower than those tests performed on $\mathrm{Si} / \mathrm{SiO}_{2}$ substrates. ${ }^{24}$ Lower carrier mobility results in large sheet resistivity of the sample, which is as high as $\sim 9.5 \mathrm{k} \Omega$ at $2 \mathrm{~K}$ and $\sim 15.1 \mathrm{k} \Omega$ at $300 \mathrm{~K}$. One possible reason behind this feature is owing to the large size of our sample (up to $\sim 20 \mathrm{~mm}^{2}$ ), where the number of graphene layers may vary spatially as suggested by a Raman spectroscopy measurement in Ref. 24. The inter-layers resistance could contribute to the overall resistance. Another possibility is that since the graphene film in this work has been transferred onto stretchable PET substrates, inevitable localized strain may also play a role as new disorder sources and hence raises the net resistivity.

As an ideal 2D system, the magnetotransport behaviors of graphene under magnetic fields with different orientations to the graphene plane are interesting but is yet to be explored. In our experiments, we investigated $\rho_{x x}$ and $\rho_{x y}$ as a function of the external field $B$ and the angular rotating angle $\theta$, where $\theta$ represents the sample position and hence $B_{z}=B \cos \theta$. When $\theta=0$, external field is normal to the graphene plane. However, in the raw experimental data $R_{C H 2}$, a difference between $R_{\mathrm{CH} 2}\left(\theta=0^{\circ}\right)$ and $R_{\mathrm{CH} 2}\left(\theta=180^{\circ}\right)$ can be clearly seen at relatively high applied field. The resistivity of the two sample positions are expected to be the same since all experimental conditions are identical except the reverse of the graphene/substrate interface. Since graphene sample is weakly attached to the PET substrate due to the transfer technique, the magnetotransport behavior should not be affected by the position because there is no time-related term involved in either the WL correction to the MR at low temperature or the MR effect at both temperatures. For the transverse resistivity, since the contribution from WL correction to $\mathrm{MR}$ in $R_{x y}$ is very small, $R_{x y}\left(\theta=90^{\circ}\right)=R_{x y}\left(\theta=270^{\circ}\right) \approx$ 0 is expected, yet neither can be seen in the experimental data. Thus, Eq. (2) could also be used for the angular dependence experimental data correction. Good agreements were also achieved between the corrected data and simulation results, which would be discussed in the following text.

Corrected curves display the angular dependence magnetotransport behaviors of our sample. As shown in Figures 4(a) and $4(\mathrm{~b})$, at the temperature of $2 \mathrm{~K}$ and $300 \mathrm{~K}$ under a few selected applied field, all observed longitudinal resistivity $\rho_{x x}$ have a form of cosine function, which contains two completed cycles when $\theta \in[0,360]$. However, at $2 \mathrm{~K}$, two additional sharp peaks are seen at $90^{\circ}$ and $270^{\circ}$. This interesting result once again clearly indicates that the total longitudinal resistivity contains two components, the negative MR due to WL and positive MR, which is a result of the unbalance force between the Hall field and Lorenz force effecting on the electrons with different energy decided by the distribution function. Therefore, at $90^{\circ}$ and $270^{\circ}$, the applied field is parallel to the graphene system, and hence its suppression on the WL effect vanishes, which brings a very large $\rho_{x x}$; as the sample is away from the parallel position, the external field weakens the negative MR due to WL and positive MR due to Lorentz force starts to dominate the $\rho_{x x}$. At $300 \mathrm{~K}$, it was concluded in the previous text that the WL effect cannot be seen at room temperature, so only magnetoresistance effect contributes the total longitudinal resistivity. For the magnetoresistance effect, $\Delta \rho / \rho(B=0) \propto B_{z}^{2} \propto B_{\text {external }} \cos ^{2} \theta \propto \cos 2 \theta$ explains the two complete cycles of the waveform function of $\rho_{x x}$. 
(a) $2 \mathrm{~K}$

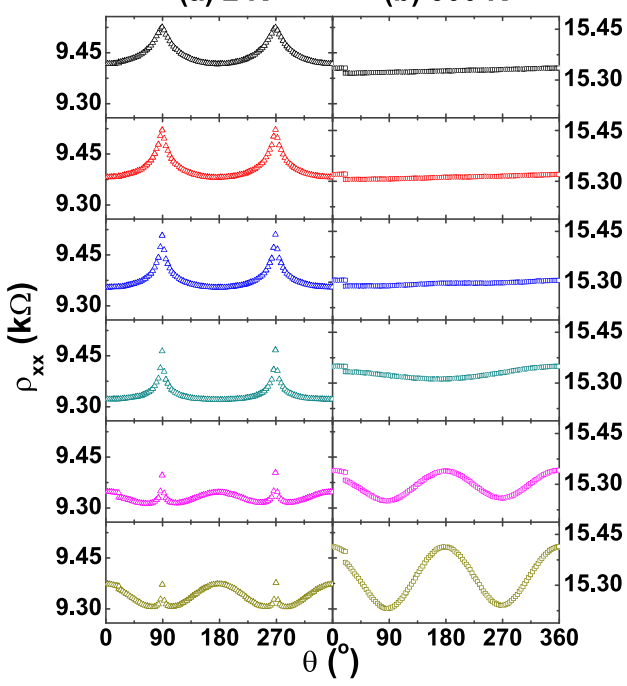

(c) $2 \mathrm{~K}$

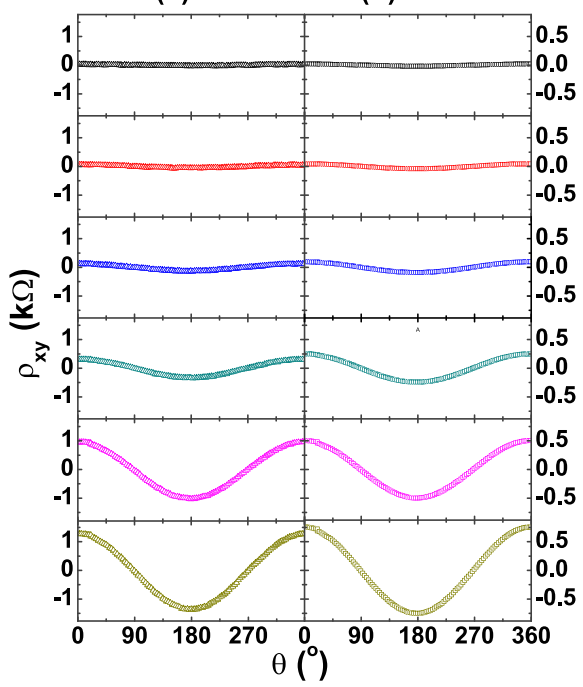

FIG. 4. (a) and (b) longitudinal resistivity $\rho_{x x}$ at $2 \mathrm{~K}$ and $300 \mathrm{~K}$. It can be seen that $\rho_{x x}$ has a form with two complete cycles. However, two additional sharp WL peaks can be seen at $90^{\circ}$ and $270^{\circ}$ at $2 \mathrm{~K}$. (c), (d) Transverse resistivity $\rho_{x y}$ at $2 \mathrm{~K}$ and $300 \mathrm{~K}$. A typical cosine waveform with the amplitude linear to the external field can be seen.
For the transverse magnetotransport behavior, as shown Figures 4(c) and 4(d), corrected transverse resistivity at both $2 \mathrm{~K}$ and $300 \mathrm{~K}$ is plotted. Since the excitation current remains unchanged, $\rho_{\text {Hall }}=R_{H} I B \propto B_{z}=B \cos \theta$, where $R_{H}$ is the Hall coefficiency. In addition, the even function component in the $\rho_{x y}$ is found to be very small. Even after the longitudinal part of the total raw $\rho_{x y}$ been subtracted from the raw data, pure transverse resistivity would still show the shape of a completed cosine function when $\theta \in[0,360]$, and the amplitude is proportional to the external field.

To obtain a thorough understanding of the experimental magneto-transport properties of the sample, we quantificationally simulated the MR as a function of the applied field $B$ and $\theta$, using Eq. (1). We use the projection of external magnetic field normal to the graphene plane $B_{z}=B \cdot \cos \theta$ replacing the $B$ in Eq. (1). In addition, the sign for the third term in the Eq. (1) is positive for the bilayered and multilayered samples, and negative for the single layered graphene. In this study, Eq. (1) with a positive sign should be mainly considered due to the minority of the single layered graphene in the film sample. Nevertheless, for the positive MC/negative MR scenario, strong intra- and inter-valley scattering result in small $\tau_{*}$ and
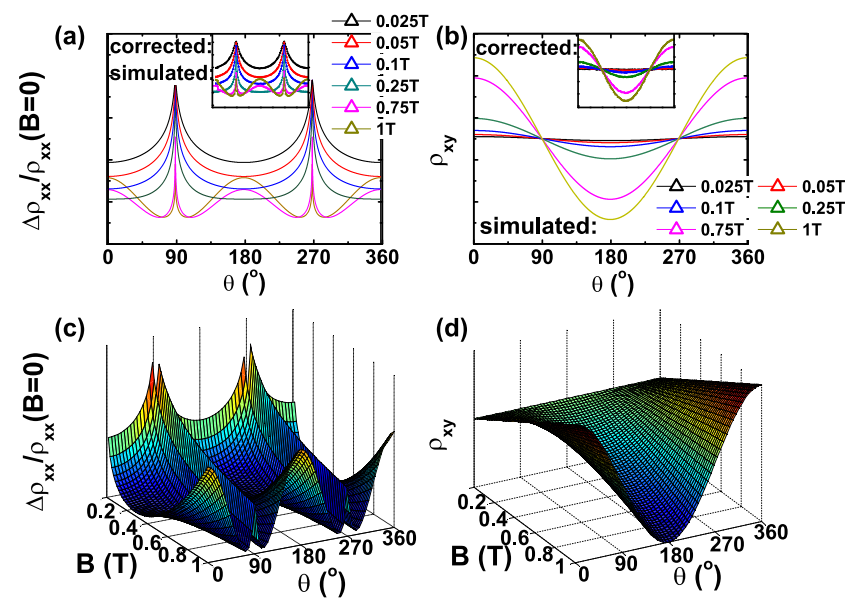

FIG. 5. (a) The simulation curves of $\Delta \rho_{x x} / \rho_{x x}(B=0)$ and (b) $\rho_{x y}$ at $2 \mathrm{~K}$ and external field at $0.025 \mathrm{~T}, 0.05 \mathrm{~T}, 0.1 \mathrm{~T}, 0.25 \mathrm{~T}, 0.75 \mathrm{~T}$, and $1 \mathrm{~T}$, respectively. Inset is the corrected experimental curves of corresponding conditions. Two sets of curves shows excellent match. (c), (d) The 3D plot of $\Delta \rho_{x x} / \rho_{x x}(B=0)$ and $\rho_{x y}$ as a function of external field $B$ and $\theta$. $\tau_{i}$; hence, the second and third term would be suppressed while the first term dominates. ${ }^{20}$ As the field increases, normal magnetoresistance effect emerges and contributes larger positive MR to the total longitudinal resistivity and thus arouses the transition. The simulation result clearly manifests two effects and yield good agreement with the experimental data.

As presented in Figures 5(a) and 5(b), MR ratio $\Delta \rho_{x x} / \rho_{x x}(B=0)$ and transverse resistivity $\rho_{x y}$ at different applied fields from simulation results and the corrected experimental data (the inset) are compared; a good agreement can also be seen in the relative values. Furthermore, since both of the longitudinal and transverse resistivity at $2 \mathrm{~K}$ contains both WL and magnetoresistance components, a threedimensional plot of $\rho_{x x}$ and $\rho_{x y}$ resistivity as a function of applied field and sample position angel is plotted, as shown in Figures 5(c) and 5(d). More details and characteristics of the interplay between WL correction to the MR and normal magnetoresistance effect can be seen, such as the lowest MR value at $1 \mathrm{~T}$ is found at $\sim 75^{\circ}, \sim 105^{\circ}, \sim 255^{\circ}$, and $\sim 285^{\circ}$, where the suppression of the MC is the weakest. The simulation result shows a good agreement with the experiment observations and confirms our analysis.

In conclusion, we have investigated the field magnitude and orientation dependence of longitudinal and transverse magnetotransport in a centimeter square graphene sheet grown by CVD method and then transferred on PET substrate. We have found that at $2 \mathrm{~K}$, the WL introduces a negative MR at low fields while Lorenz force dominates the MR at high fields at $2 \mathrm{~K}$ and all field range at $300 \mathrm{~K}$. In addition, our results suggest that weak localization may have contributes to the transverse effect at low temperature. Interesting behaviors of magnetic angle dependent resistivity have been well explained and simulated using the quantum interference theory and classical Lorentz force.

This work is supported by Australian Research Council.

${ }^{1}$ A. K. Geim, Science 324, 1530 (2009).

${ }^{2}$ K. S. Novoselov, A. K. Geim, S. V. Morozov, D. Jiang, M. I. Katsnelson, I. V. Grigorieva, S. V. Dubonos, and A. A. Firsov, Nature (London) 438, 197 (2005).

${ }^{3}$ Y. B. Zhang, Y. W. Tan, H. L. Stormer, and P. Kim, Nature (London) 438, 201 (2005). 
${ }^{4}$ M. I. Katsnelson, K. S. Novoselov, and A. K. Geim, Nat. Phys. 2, 620 (2006).

${ }^{5}$ E. McCann, K. Kechedzhi, V. I. Fal'ko, H. Suzuura, T. Ando, and B. L. Altshuler, Phys. Rev. Lett. 97, 146805 (2006).

${ }^{6}$ A. H. C. Neto, F. Guinea, N. M. R. Peres, K. S. Novoselov, and A. K. Geim, Rev. Mod. Phys. 81, 109 (2009).

${ }^{7}$ X. Wang, L. Zhi, and K. Mullen, Nano Lett. 8, 323 (2008).

${ }^{8}$ W. Y. Kim and K. S. Kim, Nat. Nano 3, 408 (2008).

${ }^{9}$ J. B. Oostinga, H. B. Heersche, X. Liu, A. F. Morpurgo, and L. M. K. Vandersypen, Nat. Mater. 7, 151 (2008).

${ }^{10}$ D. C. Elias, R. R. Nair, T. M. G. Mohiuddin, S. V. Morozov, P. Blake, M. P. Halsall, A. C. Ferrari, D. W. Boukhvalov, M. I. Katsnelson, A. K. Geim, and K. S. Novoselov, Science 323, 610 (2009).

${ }^{11}$ J. Bai, R. Cheng, F. Xiu, L. Liao, M. Wang, A. Shailos, K. L. Wang, Y. Huang, and X. Duan, Nat. Nano 5, 655 (2010).

${ }^{12}$ J. Lu, H. Zhang, W. Shi, Z. Wang, Y. Zheng, T. Zhang, N. Wang, Z. Tang, and P. Sheng, Nano Lett. 11, 2973 (2011).

${ }^{13}$ S. D. Sarma, S. Adam, E. H. Hwang, and E. Rossi, Rev. Mod. Phys. 83, 407 (2011).

${ }^{14}$ B. L. Altshuler, D. Khmel'nitzkii, A. I. Larkin, and P. A. Lee, Phys. Rev. B 22, 5142 (1980).

${ }^{15}$ S. V. Morozov, K. S. Novoselov, M. I. Katsnelson, F. Schedin, L. A. Ponomarenko, D. Jiang, and A. K. Geim, Phys. Rev. Lett. 97, 016801 (2006).

${ }^{16}$ R. V. Gorbachev, F. V. Tikhonenko, A. S. Mayorov, D. W. Horsell, and A. K. Savchenko, Phys. Rev. Lett. 98, 176805 (2007).

${ }^{17}$ K. Kechedzhi, V. I. Falko, E. McCann, and B. L. Altshuler, Phys. Rev. Lett. 98, 176806 (2007).
${ }^{18}$ Y. F. Chen, M. H. Bae, C. Chialvo, T. Dirks, A. Bezryadin, and N. Mason, Physica B 406, 785 (2011).

${ }^{19}$ F. V. Tikhonenko, D. W. Horsell, R. V. Gorbachev, and A. K. Savchenko, Phys. Rev. Lett. 100, 056802 (2008).

${ }^{20}$ F. V. Tikhonenko, A. A. Kozikov, A. K. Savchenko, and R. V. Gorbachev, Phys. Rev. Lett. 103, 226801 (2009).

${ }^{21}$ H. Cao, Q. Yu, L. A. Jauregui, J. Tian, W. Wu, Z. Liu, R. Jalilian, D. K. Benjamin, Z. Jiang, J. Bao, S. S. Pei, and Y. P. Chen, Appl. Phys. Lett. 96, 122106 (2010).

${ }^{22}$ E. Whiteway, V. Yu, J. Lefebvre, R. Gagnon, and M. Hilke, e-print arXiv: $1011.5712 \mathrm{v} 2$.

${ }^{23}$ W. A. de Heer, C. Berger, X. S. Wu, P. N. First, E. H. Conrad, X. B. Li, T. B. Li, M. Sprinkle, J. Hass, M. L. Sadowski, M. Potemski, and G. Martinez, Solid State Commun. 143, 92 (2009).

${ }^{24}$ K. S. Kim, Y. Zhao, H. Jang, S. Y. Lee, J. M. Kim, K. S. Kim, J. H. Ahn, P. Kim, J. Y. Choi, and B. H. Hong, Nature (London) 457, 706 (2009).

${ }^{25}$ A. Reina, X. Jia, J. Ho, D. Nezich, H. Son, V. Bulovic, M. S. Dresselhaus, and J. Kong, Nano Lett. 9, 30 (2008).

${ }^{26}$ H. Cao, Q. Yu, R. Colby, D. Pandey, C. S. Park, J. Lian, D. Zemlyanov, I. Childres, V. Drachev, E. A. Stach, M. Hussain, H. Li, S. S. Pei, and Y. P. Chen, J. Appl. Phys. 107, 044310 (2010).

${ }^{27}$ W. Wang, L. Chen, Z. Wang, Y. Wang, T. Li, and Y. Wang, Carbon 50, 5242 (2012).

${ }^{28}$ J. Sun, N. Lindvall, M. T. Cole, T. Wang, T. J. Booth, P. Boggild, K. B. K. Teo, J. Liu, and A. Yurgens, J. Appl. Phys. 111, 044103 (2012).

${ }^{29}$ Y. P. Liu, W. S. Lew, S. Goolaup, Z. X. Shen, L. Sun, T. J. Zhou, and S. K. Wong, Carbon 50, 2273 (2012). 